\title{
Smectic Liquid Crystals in an Anisotropic Random Environment
}

\author{
Dennis Liang* and Robert L. Leheny \\ Department of Physics and Astronomy, Johns Hopkins University, Baltimore, MD 21218, USA
}

(Dated: May 2, 2019)

\begin{abstract}
We report a high-resolution x-ray scattering study of the smectic liquid crystal octylcyanobiphenyl (8CB) confined to aligned colloidal aerosil gels. The aligned gels introduce orientational fields that promote long-range nematic order while imposing positional random fields that couple to the smectic density wave and disrupt the formation of an ordered smectic phase. At low densities of aerosil, the low-temperature scattering intensity is consistent with the presence of a topologically ordered $\mathrm{XY}$ Bragg glass phase that is predicted to form in response to such anisotropic quenched disorder. The observed features of the phase include an algebraic decay of the smectic correlations, which is truncated at large length scales due to the imperfect nematic order, and a power-law exponent that agrees closely with the universal value predicted for the XY Bragg glass. At higher aerosil densities, deviations from the XY Bragg glass form are apparent. At high temperature, the scattering intensity displays pre-transitional dynamic fluctuations associated with the destroyed nematic to smecticA transition. The fluctuations obey quasi-critical behavior over an extended range of reduced temperature. The effective critical exponents for the correlation lengths and smectic susceptibility differ systematically from those of pure $8 \mathrm{CB}$, indicating that coupling of the nematic order to the gel suppresses its role in the smectic critical behavior.
\end{abstract}

PACS numbers: 61.30.Pq, 61.30.Eb, 64.70.Md, 61.10.Eq

\section{INTRODUCTION}

The effect of impurities and quenched disorder in condensed matter represents an important problem both because of the ways in which disorder can change fundamental properties of a system and because any real material will inevitably possess imperfections. Studies have shown dramatic consequences of disorder, such as the destruction of ordered phases and the introduction of new exotic ones, deviation from the universal behavior around transition points, and sometimes even the enhancement of useful material properties. Liquid crystals, due to their soft elasticity, sensitivity to surface interactions, and experimental accessibility, provide an excellent opportunity for studying the effects of quenched disorder, which can be introduced experimentally through confinement of the liquid crystal to random porous media. A particular focus in this area has been the study of smectic liquid crystals in silica gels. In a detailed theoretical treatment of smectics in such random environments, Radzihovsky, Toner, and coworkers identified two forms of random-field disorder that the confinement introduces to the smectic - random orientational fields that couple to the nematic director and random positional fields that couple to the smectic density wave [1, 2, $, 3,4,[5]$. One conclusion of their study was that the smectic phase is unstable to arbitrarily weak random-field disorder, consistent with expectations for a phase that breaks a continuous symmetry in three dimensions. Experimentally, considerable work has been dedicated toward investigating smectics in two types of silica gels, chemically rigid

\footnotetext{
* Present address: Intense Pulsed Neutron Source Division, Argonne National Laboratory, Argonne, IL 60439
}

aerogels $6,77,8,9]$ and colloidal gels formed from aerosil particles 10, 11, 12, 13, 14, 15, 16, 17, 18, 19, 20, 21, 22, $23,24,25,26,27,28,29]$. The chemical compositions and structures of the two gels are very similar, but the hydogen-bonded aerosil gels have the potential to access a weaker regime of disorder. Utilizing a wide range of probes, experiments on smectics confined to aerogels and aerosil gels have detailed the consequences of the confinement for the nematic and smectic phases, supporting the general picture that the gels impose random-field disorder that destroys the nematic to smectic transition.

Among the predictions for smectics confined to gels is the potential formation of topologically ordered, "Bragg glass" phases that are distinct from the high-temperature nematic phase by the absence of unbound dislocation loops. Considerable evidence from theory and simulation supports the existence of a topologically ordered state with algebraic decay of correlations in the threedimensional (3D) random field XY model at low temperature 30, 31, 32]. (However, a recent argument based on the functional renormalization group approach has questioned the existence theoretically of such a phase in 3D XY systems [33].) Since the nematic to smectic-A (NSmA) transition breaks 3D XY symmetry, one might hence expect that smectics confined to gels would be good candidates for realizing such an "XY Bragg glass" phase. Considering this possibility theoretically, Radzihovsky and Toner have concluded that the orientational random fields coupling to the nematic order and the soft elasticity of the smectic make smectics confined to gels distinct from standard random field XY systems. Instead, they have introduced the possibility that a "smectic Bragg glass" phase, which has short range correlations and is qualitatively different from the XY Bragg glass, might form in smectics confined to gels [1]. However, the theoretical argument for the stability of the smectic 
Bragg glass is inconclusive, and experimental evidence for the phase in smectics confined by silica gels has been contradictory [6, 12, 13, 27]. Jacobsen, Saunders, et al. have further argued that smectics confined to uniaxially strained gels should fall into the universality class of random field XY systems and hence should form an "XY Bragg glass" for sufficiently weak disorder [4, 5, 34]. The key difference between isotropic gels and strained gels is the effect of the orientational fields. Specifically, if the strain alters the distribution of orientational fields, causing them to suppress nematic fluctuations at large length scales, then the smectic in strained gels should theoretically exhibit an XY Bragg glass, provided the positional random fields are sufficiently weak. Recent additional theoretical predictions regarding the smecticA to smectic-C transition under confinement in strained gels has highlighted the potential for smectics in such an anisotropic environment to display exotic phase behavior [34].

In an effort to understand the separate effects of orientational random fields and positional random fields on the N-SmA transition and to test for the presence of an $\mathrm{XY}$ Bragg glass phase, we have conducted an x-ray scattering study of smectic octylcyanobiphenyl (8CB) confined to aligned aerosil gels. The structure of these gels dramatically alters the nature of the orientational fields, converting the random distribution found in isotropic gels into a sharply anisotropic distribution that macroscopically orders the nematic director. This anisotropy, or "soft axis", makes the gels a faithful experimental realization of uniaxially strained systems considered by Jacobsen, Saunders, et al. In this paper, we report an analysis of the x-ray scattering results that indicates the presence of an XY Bragg glass at low temperature for low densities of aligned aerosil. The signature feature of the XY Bragg glass is a power-law divergent smectic scattering peak with a universal exponent. We note that in a preliminary report on our x-ray studies [14], we concluded that $8 \mathrm{CB}$ confined to aligned aerosil gels did not exhibit such characteristics of the XY Bragg glass phase. As we explain below, by adopting a physically better-motivated description of the behavior of the thermal critical fluctuations in smectics confined to aerosil gels [28], we find that the previous analysis is not satisfactory in accounting for the smectic correlations at low temperature. Instead, when a finite nematic domain size is incorporated into the analysis to reflect the imperfect nematic order in the aligned gels, comparisons between the x-ray scattering results for low densities of aerosil and the predictions for an XY Bragg glass show excellent agreement. A discussion of these findings is given in Sec. III below.

Another important benefit of the macroscopic alignment of the nematic director by the anisotropic gels is the pronounced enhancement in signal-to-background in $\mathrm{x}$-ray measurements relative to measurements on smectics in isotropic gels. Taking advantage of this enhancement, we further provide in Sec. IV a detailed study of the smectic correlations at high temperature, which we analyze in terms of dynamic fluctuations that grow on approaching the critical region of the destroyed N-SmA transition. This analysis shows that the correlations, as characterized by effective critical exponents for the correlation lengths and susceptibility, differ from those of pure $8 \mathrm{CB}$ even when the correlation lengths are considerably smaller than the gel pore size, providing evidence that the presence of quenched disorder alters the coupling between nematic and smectic order and its influence over smectic critical behavior. When combined with similar results from smectics confined to isotropic gels for effective critical exponents for the specific heat and smectic order parameter [12, 13, 28], these findings provide a comprehensive picture of an evolution in the N-SmA critical fluctuations toward conventional 3D XY behavior with increasing quenched disorder.

\section{SAMPLE PREPARATION AND CHARACTERIZATION}

Pure $8 \mathrm{CB}$ undergoes an isotropic to nematic transition at $T_{N I}=313.98 \mathrm{~K}$ and a N-SmA transition at $T_{N A}$ $=306.97 \mathrm{~K}[19]$. Confinement of the liquid crystal to aligned aerosil gels was accomplished through a procedure described previously [14]. Type 300 hydrophilic aerosil (DeGussa Corp.) was dried under vacuum at 393 $\mathrm{K}$ for 24 hours. The dried powder along with appropriate quantities of $8 \mathrm{CB}$ (Frinton Laboratories, Inc.) was dissolved in high-purity acetone, and the mixtures were sonicated for at least two hours. The solutions were then heated to $318 \mathrm{~K}$ to evaporate the solvent slowly. After no visible trace of acetone remained, the samples were heated to $338 \mathrm{~K}$ under vacuum. The resulting composites consisted of $8 \mathrm{CB}$ confined to isotropic fractal aerosil gels like those of previous studies [10, 11, 12, 13, 14, 16, 17]. To create anisotropic gels, we placed each sample in a 2 Tesla magnetic field and cycled the temperature between $308 \mathrm{~K}$ (nematic phase) and $318 \mathrm{~K}$ (isotropic phase) at least 100 times. Due to the magnetic anisotropy of nematic $8 \mathrm{CB}$, the director tends to align parallel to the magnetic field, in competition with the random orientational fields created by the gel. Due to the elasticity of the nematic and the compliance of the gel, this competition results in restructuring of the gel to accommodate the magnetic anisotropy. As a result, the gels acquire a structure in which the orientational fields are no longer random but on average orient parallel to the aligning direction. Since the gel continues to have a random structure positionally, the positional fields that couple to the smectic density wave remain random. Samples were prepared in this manner with aerosil densities ranging from $\rho_{s}=0.027 \mathrm{~g} \mathrm{sil} / \mathrm{cm}^{3} 8 \mathrm{CB}$, which is just above the gelation threshold, to $\rho_{s}=0.10 \mathrm{~g} \mathrm{sil} / \mathrm{cm}^{3} 8 \mathrm{CB}$, which is the upper limit above which efforts to align the gels were ineffectual.

The smectic correlations that form in this anisotropic random environment were studied through high- 


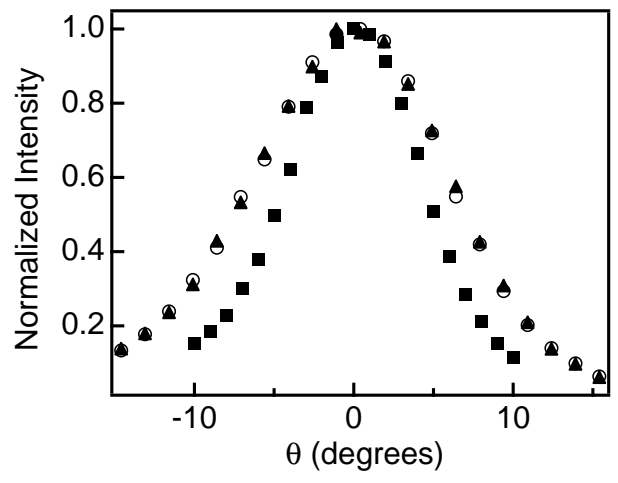

FIG. 1: Normalized scattering intensity of the smectic peak at fixed wave vector magnitude $\left(q \approx 0.2 \AA^{-1}\right)$ as a function of sample orientation for $8 \mathrm{CB}$ confined by an aligned aerosil gel with $\rho_{s}=0.027 \mathrm{~g} / \mathrm{cm}^{3}$ (solid squares) and $0.10 \mathrm{~g} / \mathrm{cm}^{3}$ (open circles and solid triangles) at $298.15 \mathrm{~K}$. The two scattering intensities for $\rho_{s}=0.10 \mathrm{~g} / \mathrm{cm}^{3}$ correspond to measurements before (open circles) and after (solid triangles) annealing the sample in the isotropic phase of $8 \mathrm{CB}$.

resolution x-ray scattering. The experiments were conducted at the X22A beam line of the National Synchrotron Light Source. The beam line is equipped with a $\mathrm{Ge}(111)$ monochromator to select a beam of $10 \mathrm{keV} \mathrm{x}$ rays. The measurements were performed in transmission geometry. The beam size was approximately $1 \mathrm{~mm} \times$ $1 \mathrm{~mm}$, and the sample thickness was approximately 1.5 $\mathrm{mm}$ to match the attenuation length of the $10 \mathrm{keV}$ x-rays. A triple-bounce $\mathrm{Si}(111)$ channel-cut analyzer crystal was positioned between the sample and a scintillation point detector to achieve high wave-vector resolution.

To determine the degree of anisotropy in the orientational fields coupling to the liquid crystal, we characterized the mosaic spread of the smectic layer normal by "rocking curve" measurements through the smectic scattering peak. Figure 1 shows mosaic scans for $\rho_{s}=0.027$ and $0.10 \mathrm{~g} / \mathrm{cm}^{3}$ at $\mathrm{T}=298.1 \mathrm{~K}$, several degrees below $T_{N A}$. The smectic scattering displays strong azimuthal anisotropy, indicating a narrow distribution of smectic layer-normal orientations. The alignment is slightly better (the peak is narrower) for lower density gels, consistent with the gels' increased ability to resist restructuring with increasing density due to greater in mechanical strength [13, 29]. We note these measurements were performed several weeks after removal of the magnetic field used to align the gels; thus, the observed macroscopic alignment is a consequence of the gel and not the external field. As also shown in Fig. 1, the quality of the nematic alignment displays negligible change in response to temperature excursions into the isotropic phase, demonstrating that the anisotropic gel structure is stable against thermal fluctuations and sufficiently robust to re-align the nematic director on subsequent cooling. Indeed, samples are observed to remain aligned after repeated heating into the isotropic phase and after storage for several months. Thus, the random orientational fields of the isotropic gel have been converted into fields with long-range order that align the nematic director macroscopically.

\section{SMECTIC CORRELATIONS: EVIDENCE FOR AN XY BRAGG GLASS}

Figure 2(a) displays the low-temperature x-ray scattering intensity for $\rho_{s}=0.027 \mathrm{~g} / \mathrm{cm}^{3}$ as a function of wave vector parallel to the alignment direction (i.e., the direction of the magnetic field used to prepare the gel), $q_{z}$, and perpendicular to it, $q_{x}$. In the alignment direction the intensity displays a pronounced peak corresponding to the smectic scattering, while in the perpendicular direction the intensity shows no evidence of smectic scattering, consistent with the strong anisotropy implied by Fig. 1. We ascribe the scattering intensity along $q_{x}$ entirely to the gel structure and note that an essentially identical background intensity contributes to the scattering along $q_{z}$. In order to isolate the smectic scattering from this background, we subtract the intensity along $q_{x}$ from the intensity along $q_{z}$. This procedure assumes that the anisotropy in the gel structure does not affect the scattering from the gel in the wave-vector range of the smectic peak. As described previously, small angle scattering measurements support this assumption [14]. Nevertheless, the observed smectic signal greatly exceeds this background contribution, by a factor of more than a factor of $10^{4}$ in Fig. 2(a), and this strong signal-tobackground, which results from the azimuthal focusing by the aligned orientational fields, enables the detailed examination of the smectic correlations described below. Figure 2(b) displays the results for the scattering intensity along $q_{z}$ from Fig. 2(a) with the background subtracted. Also in the figure is the resolution function determined from the profile of the direct x-ray beam. The inset to Fig. 2(b) compares the scattering to the resolution in the region of the peak. The peak is clearly broader than the resolution even for this lowest aerosil density at low temperature.

As a first effort to characterize the smectic order, we consider a correlation function based on the randomfield model that has been used previously to describe the short-range smectic correlations observed under confinement in isotropic aerosil gels [10, 12, 26, 27]. Specifically, we model the smectic peak with the two-component line shape

$$
\begin{array}{r}
I(\mathbf{q})=\frac{\sigma_{1}}{1+\left(q_{\|}-q_{0}\right)^{2} \xi_{1 \|}^{2}+q_{\perp}^{2} \xi_{1 \perp}^{2}+c_{1} q_{\perp}^{4} \xi_{1 \perp}^{4}} \\
+\frac{a_{2}\left(\xi_{2 \|} \xi_{2 \perp}^{2}\right)}{\left(1+\left(q_{\|}-q_{0}\right)^{2} \xi_{2 \|}^{2}+q_{\perp}^{2} \xi_{2 \perp}^{2}+c_{2} q_{\perp}^{4} \xi_{2 \perp}^{4}\right)^{2}}
\end{array}
$$

where $q_{\|}$and $q_{\perp}$ are the wave vectors parallel and perpendicular to the smectic layer normal, respectively, and $q_{0}$ is the ordering wave vector. The first term in $I(\mathbf{q})$ is an anisotropic Lorentzian with fourth-order correction 


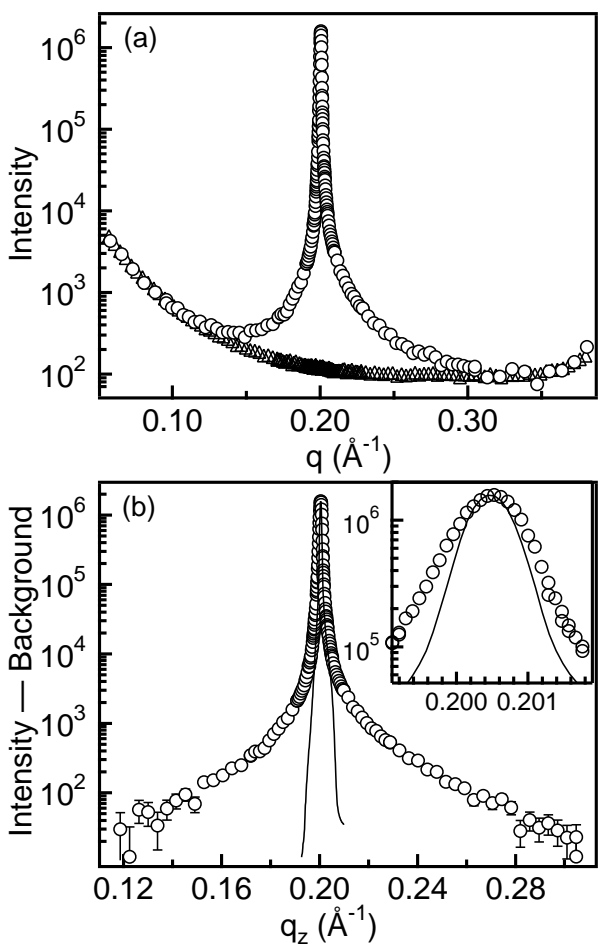

FIG. 2: (a) X-ray scattering intensity for $8 \mathrm{CB}$ confined by an aligned aerosil gel with $\rho_{S}=0.027 \mathrm{~g} / \mathrm{cm}^{3}$ at $296.15 \mathrm{~K}$ as a function of wave vector parallel to the gel's alignment direction, $q_{z}$ (circles) and perpendicular to this direction, $q_{x}$ (triangles). (b) Scattering intensity along $q_{z}$ with the intensity along $q_{x}$ subtracted to isolate the smectic scattering. The solid line in (b) is the instrumental resolution obtained from the incident beam profile. The inset to (b) shows the peak region on an expanded scale.

that describes critical dynamic fluctuations on approaching the N-SmA transition in pure liquid crystals. $\sigma_{1}$ is the smectic susceptibility, and $\xi_{1 \|}$ and $\xi_{1 \perp}$ are the correlation lengths of the fluctuations in the directions parallel and perpendicular to the nematic director, respectively. The second term, which has a shape that is proportional to the square of the thermal fluctuation term, describes static short-range fluctuations due to the random fields, which are characterized by the correlation lengths $\xi_{2 \|}$ and $\xi_{2 \perp}$. Previous studies of smectics in isotropic aerosil gels have found that the correlations display two temperature regimes. At low temperature, $a_{2}>0$, and the amplitude of the static fluctuation term rises from zero with decreasing temperature. At higher temperature, $a_{2}=0$, and the scattering profile is similar to that of the nematic phase of pure $8 \mathrm{CB}$, where pre-transitional smectic critical fluctuations dominate [12, 27, 28].

Figure 3 shows the results of fits to Eq. (1) with $a_{2}=0$ at two temperatures in this high-temperature regime for $8 \mathrm{CB}$ confined by an aligned gel with $\rho_{s}=0.10 \mathrm{~g} / \mathrm{cm}^{3}$. To perform fits to Eq. (1), we integrate numerically over the distribution of layer normal orientations, as determined

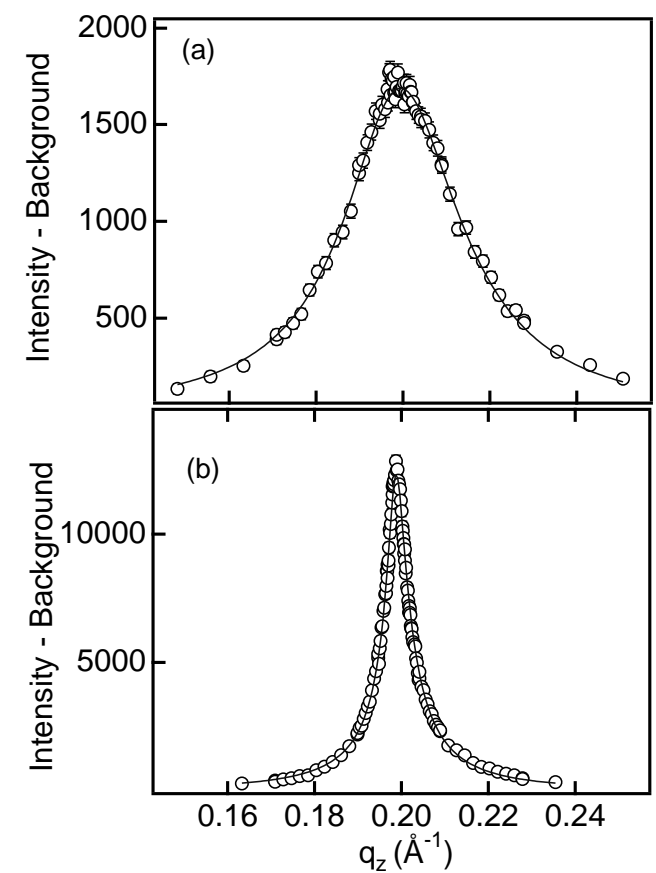

FIG. 3: Background-subtracted scattering intensity of the smectic peak for $8 \mathrm{CB}$ confined to an aligned aerosil gel with $\rho_{s}=0.10 \mathrm{~g} / \mathrm{cm}^{3}$ at (a) $309 \mathrm{~K}$ and (b) $306 \mathrm{~K}$. The solid lines are the results of fits to Eq. (1) with $a_{2}=0$.

by rocking curves like those shown in Fig. 1. and convolve with the instrumental resolution. In this analysis $\xi_{1 \|}$ and $\xi_{1 \perp}$ are treated as independent parameters, while the amplitude of the fourth-order correction term, $c$, is treated as a function of $\xi_{1 \perp}$, with $c\left(\xi_{1 \perp}\right)$ set by the behavior in pure $8 \mathrm{CB}$ [35, 36, 37]. (Allowing $c$ to vary as an additional free parameter leads to significant scatter in the fit parameters.) As Fig. 3 illustrates, the dynamic term in Eq. (1) provides an excellent description of pre- transitional smectic fluctuations in $8 \mathrm{CB}$ confined to aligned aerosil gels at high temperatures. This agreement allows us to study in detail the pseudo-critical behavior of $8 \mathrm{CB}$ with anisotropic quenched disorder, as described in Sec. IV below.

Turning to low temperature, the dynamic fluctuation term in Eq. (1) alone cannot describe the measured lineshape. As mentioned above, previous analysis of the smectic correlations under confinement in isotropic aerosil gels [10, 12, 26, 27] have modeled the low-temperature scattering by including the contribution in Eq. (1) from static fluctuations $\left(a_{2}>0\right)$. In most cases, this analysis has assumed that $\xi_{2 \|}=\xi_{1 \|}$ and $\xi_{2 \perp}=\xi_{1 \perp}$; that is, the correlation lengths in the static and dynamic terms were assumed equal. Typically, this approach provided good agreement with the measured line shapes. Indeed, in a preliminary report on $8 \mathrm{CB}$ confined to aligned aerosil gels, we found that Eq. (1) with $\xi_{2 \|}=\xi_{1 \|}$ and $\xi_{2 \perp}=\xi_{1 \perp}$ described the data accurately [14]. However, this agreement does not neces- 
sarily indicate that the analysis is capturing the correct physical picture. As pointed out recently by Larochelle et al., the results of such analysis lead to trends in the smectic susceptibility and correlation lengths that are inconsistent with calorimetric studies of smectics confined to aerosil gels [28]. Calorimetry measurements on $8 \mathrm{CB}$ in isotropic aerosil gels at low density $\left(\rho_{s}<0.10 \mathrm{~g} / \mathrm{cm}^{3}\right)$ show a sharp peak in the heat capacity, with power-law temperature dependence resembling pre-transitional critical behavior extending over an extended range of reduced temperature both above and below an effective transition temperature $T^{*}$ [16]. This behavior would suggest that $\xi_{1 \|}, \xi_{1 \perp}$, and $\sigma_{1}$ should similarly decay as a power law as a function of reduced temperature away from $\mathrm{T}^{*}$. As discussed in Sec. IV, for $\mathrm{T}>\mathrm{T}^{*}$ the smectic fluctuations in $8 \mathrm{CB}$ confined to aligned aerosil gels indeed show a quasi-critical increase toward $\mathrm{T}^{*}$. However, analysis of the x-ray results at low temperature using Eq. (1) with $\xi_{2 \|}=\xi_{1 \|}$ and $\xi_{2 \perp}=\xi_{1 \perp}$ leads to correlation length and susceptibility values that remain large away from $\mathrm{T}^{*}$. For example, $\xi_{\|} \approx 3000 \AA$ at $\mathrm{T}^{*}-\mathrm{T}=9 \mathrm{~K}$ for $8 \mathrm{CB}$ confined to an aligned gel with $\rho_{s}=0.10 \mathrm{~g} / \mathrm{cm}^{3}$ [14], inconsistent with pseudo-critical behavior. Such large dynamic fluctuations persisting to low temperature seem very unlikely. However, if we restrict the dynamic fluctuations to follow a more plausible temperature dependence, we find that Eq. (1) cannot describe the x-ray lineshapes for $8 \mathrm{CB}$ in aligned gels at low temperature. Rather, as we explain below, the lineshape predicted for the XY Bragg glass with an appropriate cut-off for a finite nematic domain size can very accurately describe the measured lineshapes for low densities of aligned aerosil.

To examine the situation more closely, we display in Figs. 4(a) and 4(b) log-log plots of the scattering intensity versus $\left(q_{z}-q_{0}\right)$ at various temperatures for $\rho_{s}=0.042$ $\mathrm{g} / \mathrm{cm}^{3}$. As described in Sec. IV, the effective transition temperature for $\rho_{s}=0.042 \mathrm{~g} / \mathrm{cm}^{3}$ is $\mathrm{T}^{*}=305.0 \mathrm{~K}$. As seen in Fig. $4(\mathrm{a})$, the intensities at temperatures above $\mathrm{T}^{*}$ overlap at large $\left(q_{z}-q_{0}\right)$. This collapse indicates that the intensities away from the peak share the same wave-vector dependence, specifically that of the critical fluctuation term in Eq. (1), and further that the ratio $\xi_{1 \|}^{2} / \sigma_{1}$ is a constant independent of temperature (implying the critical exponent $(2-\eta) \approx 2)$. Similarly, for the two temperatures just below $\mathrm{T}^{*}$ shown in Fig. 4 (a), 303.9 $\mathrm{K}$ and $304.6 \mathrm{~K}$, the intensities at large $\left(q_{z}-q_{0}\right)$ collapse onto the data for $\mathrm{T}>\mathrm{T}^{*}$. This collapse indicates that critical fluctuations continue to dominate the intensity at large $\left(q_{z}-q_{0}\right)$ at temperatures near but below $\mathrm{T}^{*}$. This finding is not surprising since one would expect to have large critical fluctuations in the vicinity of a true critical point. However, for lower temperature, the situation becomes more complicated. Plotted in Fig. 4(b) is the intensity at $300.1 \mathrm{~K}$, approximately $4.9 \mathrm{~K}$ below $\mathrm{T}^{*}$, along with the intensity at $309.1 \mathrm{~K}$, approximately 4.1 $\mathrm{K}$ above $\mathrm{T}^{*}$. Not only does the low-temperature curve fail to collapse onto the high-temperature curve at large $\left(q_{z}-q_{0}\right)$, but it exhibits considerable excess intensity at

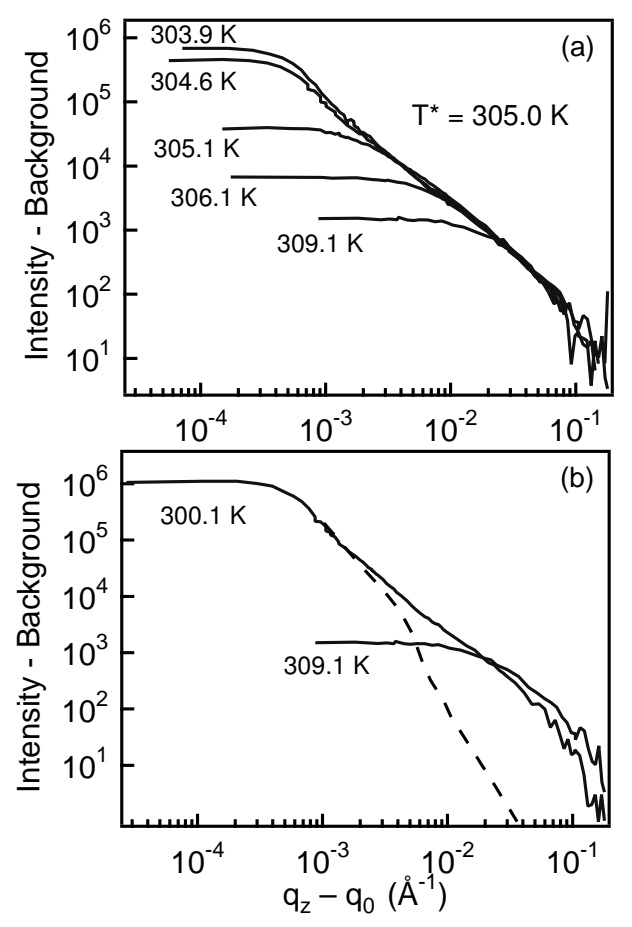

FIG. 4: Background-subtracted scattering intensity at various temperatures as a function of the difference in wave vector from the ordering wave vector, $q_{0}$, for $\rho_{s}=0.042 \mathrm{~g} / \mathrm{cm}^{3}$. (a) intensities at several temperatures ranging from just below $\mathrm{T}^{*}$ $=305.0 \mathrm{~K}$ to several degrees above $\mathrm{T}^{*}$. (b) intensities at two temperatures, $300.1 \mathrm{~K}$ and $309.1 \mathrm{~K}$, that are approximately the same difference in temperature from $\mathrm{T}^{*}$. The dashed line in (b) is the result of a fitting the intensity at $300.1 \mathrm{~K}$ in the peak region $\left(\left(q_{z}-q_{0}\right)<10^{-3} \AA^{-1}\right)$ to the second term in Eq. (1) $\left(\sigma_{1}=0\right)$ representing static short-range order.

smaller $\left(q_{z}-q_{0}\right)$ that cannot be accounted for by either term in Eq. (11). Specifically, the dashed line in Fig. 4(b) shows the result of fitting the low-temperature intensity in the peak region to the static term of Eq. (1) alone $\left(\sigma_{1}=0\right)$. While the fit agrees adequately with the measured intensity in the immediate vicinity of the peak, it deviates significantly at larger $\left(q_{z}-q_{0}\right)$. A comparison with the high-temperature intensity demonstrates that the dynamic term in Eq. (1) cannot account for these deviations. Since the two intensities plotted in Fig. 4(b) are for temperatures that are approximately the same difference in temperature from $\mathrm{T}^{*}$, they correspond to approximately the same reduced temperature. Hence, the pre-transitional critical fluctuations at the two temperatures should be similar (assuming the amplitude ratios for the correlation lengths and susceptibility are of order one). For scattering from such critical fluctuations, a power law in the intensity should be observed only for $\left(q_{z}-q_{0}\right)>1 / \xi_{1 \|}$. An inspection of the line shape for $309.1 \mathrm{~K}$ indicates that for this reduced temperature this asymptotic range is reached only for $\left(q_{z}-q_{0}\right)>4 \times 10^{-2}$ $\AA^{-1}$. The extension to lower $\left(q_{z}-q_{0}\right)$ of the power-law 
behavior at $300.1 \mathrm{~K}$ thus indicates that the scattering intensity is inconsistent with the small dynamic correlation lengths expected at a temperature so far below $\mathrm{T}^{*}$. In essence, the constraint used in the previous analysis [14] that $\xi_{2 \|}=\xi_{1 \|}$ and $\xi_{2 \perp}=\xi_{1 \perp}$ led to artificially large values of $\xi_{1 \|}$ and $\xi_{1 \perp}$ at low temperature that fortuitously approximated the measured line shape but that on further inspection cannot be justified as credible behavior for critical fluctuations.

The extended power-law tails in the low-temperature scattering peak instead suggest the possible presence of the XY Bragg glass phase. As mentioned above, the XY Bragg glass is ideally characterized by a power- law divergent smectic scattering peak with a universal power-law exponent. As noted by the inset to Fig. 2(a), the measured scattering peaks are appreciably broader than the instrumental resolution, which precludes such power-law divergence extending to the lowest $\left(q_{z}-q_{0}\right)$. However, we argue that this discrepancy could be a consequence of a large length scale cut-off introduced by the liquid crystal ordering in the aligned gels. Specifically, while the mosaic scans in Fig. 1 indicate long-range nematic order, they also show an appreciable spread in the smectic layer-normal orientations about the aligning direction. This spread indicates spatial variations in the smectic layer normal that are likely set by the length scale characterizing the nematic order. In other words, the liquid crystal in the aligned gel has finite-size nematic domains like in the isotropic gels 38], the difference being that the domains are not randomly oriented but rather have a distribution of orientations clustered around the aligning direction. Nevertheless, smectic order cannot persist across two misaligned domains, leading directly to a cutoff in the smectic correlations at low $\left(q_{z}-q_{0}\right)$.

Taking such an effect into account, we model the scattering peak at low temperature with a form

$$
I(\mathbf{q})=P(\mathbf{q})+H(\mathbf{q})
$$

where $P(\mathbf{q})$ is the XY Bragg glass correlation function truncated at small $\left(q_{z}-q_{0}\right)$, and $H(\mathbf{q})$ accounts for the finite-size effect within a Gaussian approximation [39, 40]. The XY Bragg glass form is given by [5, 34]

$$
P(\mathbf{q})=\left\{\begin{array}{c}
C\left(\left(q_{\|}-q_{0}\right)^{2}+\alpha q_{\perp}^{2}\right)^{-\delta / 2} \\
\quad \text { if }\left(\left(q_{\|}-q_{0}\right)^{2}+\alpha q_{\perp}^{2}\right)>1 / L \\
C L^{\delta} \quad \text { if }\left(\left(q_{\|}-q_{0}\right)^{2}+\alpha q_{\perp}^{2}\right)<1 / L
\end{array}\right.
$$

where $\delta$ is a universal, temperature-independent exponent predicted to be $\delta=2.45$ [5, 34], $C$ sets the overall amplitude, $\alpha$ is a non-universal constant that is expected to be of order unity [34], and $L$ is the size scale of the nematic domains. The finite-size correction has the form [40]

$$
H(\mathbf{q})=A e^{-L^{2}\left(\left(q_{\|}-q_{0}\right)^{2}+\alpha q_{\perp}^{2}\right) / 4 \pi}
$$

where the amplitude $A$ is related to $C$ such that at wave vectors $\left(\left(q_{\|}-q_{0}\right)^{2}+\alpha q_{\perp}^{2}\right)<1 / L$ the Gaussian form of

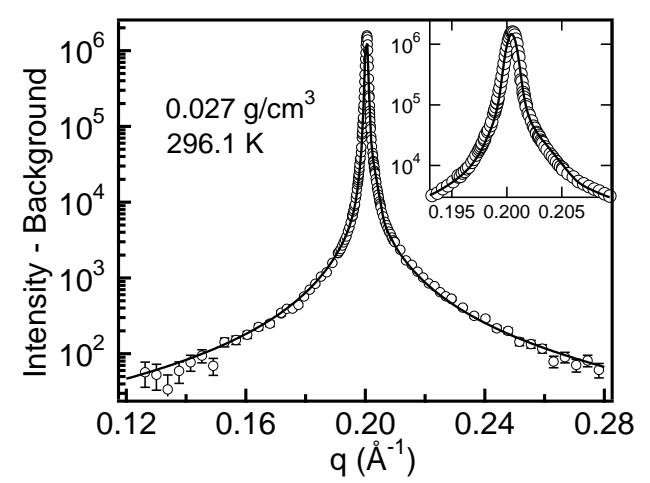

FIG. 5: Comparison between the background-subtracted scattering intensity as a function of wave vector for $\rho_{s}=0.027$ $\mathrm{g} / \mathrm{cm}^{3}$ at $296.1 \mathrm{~K}$ and the form predicted for an XY Bragg glass with a truncation in the correlations at large length scales. The circles are the measured intensity, and the solid line is the result of a fit to Eq. (2). The inset shows a comparison between the scattering intensity and the fit result in the peak region on an expanded scale. The XY Bragg glass form agrees closely with the measured intensity over the full range of wave vectors.

$H(\mathbf{q})$ dictates the line shape, while for larger wave vectors it has negligible effect on the line shape [39]. To perform fits to Eq. (2), we again integrate numerically over the distribution of layer normal orientations, as determined by rocking curves like those shown in Fig. 1, and convolve with the instrumental resolution. Figure 5 shows the result of a fit to Eq. (2) for $\rho_{s}=0.027 \mathrm{~g} / \mathrm{cm}^{3}$ at $\mathrm{T}=296.1 \mathrm{~K}$, the lowest measurement temperature. The figure displays the scattering intensity over the full range of wave vectors, while the inset shows the peak region on an expanded scale. The agreement between the measured line shape and the XY Bragg glass form is essentially perfect. Good agreement is similarly found for $\rho_{s}=0.42 \mathrm{~g} / \mathrm{cm}^{3}$, as illustrated by Fig. 6 which shows the result of a fit using Eq. (2) to the scattering intensity at $\mathrm{T}=298.1 \mathrm{~K}$ for this aerosil density. The fits for both densities give $L \approx 1 \mu \mathrm{m}$, in reasonable agreement with a value expected based on the nematic correlation lengths measured in isotropic aerosil gels [38]. The anisotropy factor is found to be $\alpha \approx 0.15$ in the two cases. For larger densities of aligned aerosil, the XY Bragg glass form is less successful in describing the measured line shape, as illustrated by Fig. 7 which shows the result of a fit using Eq. (2) to the low- temperature scattering intensity for $\rho_{s}=0.10 \mathrm{~g} / \mathrm{cm}^{3}$. We interpret this discrepancy as an indication that the larger aerosil densities impose positional disorder that is too strong for the XY Bragg glass to be stable.

For the lower aerosil densities, the close agreement with the XY Bragg glass form extends over a range of temperatures below $\mathrm{T}^{*}$. However, at temperatures sufficiently close to $\mathrm{T}^{*}$, contributions to the scattering from smectic critical fluctuations become appreciable. These contribu- 


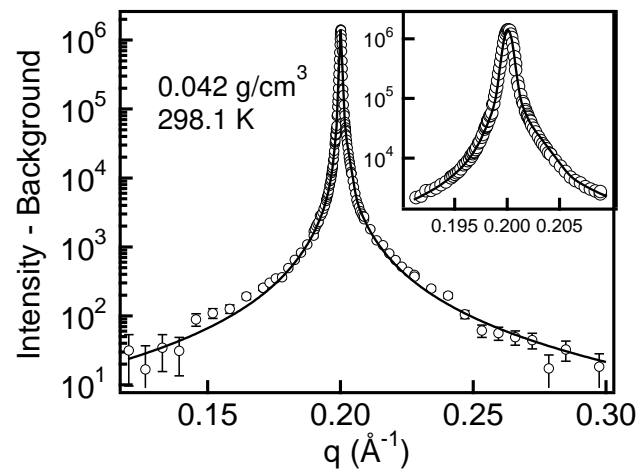

FIG. 6: Comparison between the background-subtracted scattering intensity as a function of wave vector for $\rho_{s}=0.042$ $\mathrm{g} / \mathrm{cm}^{3}$ at $298.1 \mathrm{~K}$ and the form predicted for an XY Bragg glass with a truncation in the correlations at large length scales. The circles are the measured intensity, and the solid line is the result of a fit to Eq. (2). The inset shows a comparison between the scattering intensity and the fit result in the peak region on an expanded scale. The XY Bragg glass form agrees closely with the measured intensity over the full range of wave vectors.

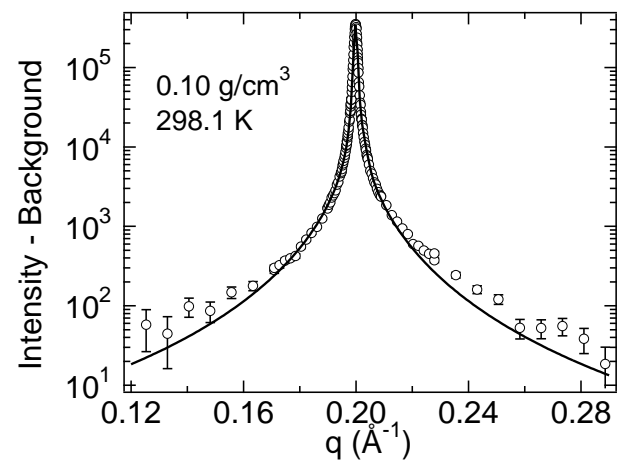

FIG. 7: Comparison between the background-subtracted scattering intensity as a function of wave vector for $\rho_{s}=0.10$ $\mathrm{g} / \mathrm{cm}^{3}$ at $298.1 \mathrm{~K}$ and the form predicted for an XY Bragg glass with a truncation in the correlations at large length scales. The circles are the measured intensity, and the solid line is the result of a fit to Eq. (2). Deviations between the XY Bragg glass form and the measured intensity are apparent at large wave vectors.

tions are apparent in the two temperatures slightly below $\mathrm{T}^{*}$ included in Fig. 4(a) where both smectic critical fluctuations at large $\left(q_{z}-q_{0}\right)$ and additional contributions at smaller wave vector are apparent. Efforts to account for the contributions from dynamic fluctuations in this temperature region by adding a term to Eq. (2) are successful in the sense that good agreement with the measured scattering intensity can be achieved. However, this analysis leads to large uncertainties in the fit parameters; therefore, we will not discuss this temperature region and will restrict our comparison to the XY Bragg glass form

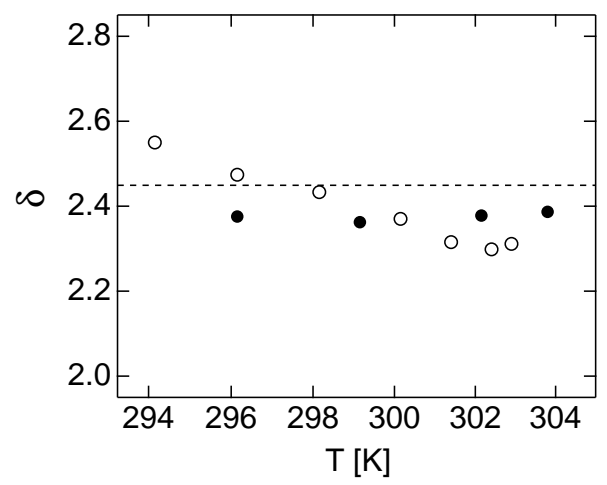

FIG. 8: Power-law exponent $\delta$ for the XY Bragg glass as a function of temperature for $8 \mathrm{CB}$ confined to aligned aerosil gels with densities $\rho_{s}=0.027 \mathrm{~g} / \mathrm{cm}^{3}$ (solid) and $0.042 \mathrm{~g} / \mathrm{cm}^{3}$ (open). The dashed line is the universal value for the exponent, $\delta=2.45$, predicted theoretically [5, 34].

to lower temperatures, where the dynamic fluctuations can be safely neglected. Figure 8 displays the values of the power-law exponent $\delta$ extracted from fits over this low-temperature region for $\rho_{s}=0.027$ and $0.042 \mathrm{~g} / \mathrm{cm}^{3}$. The measured exponents for both densities are remarkably close to the universal value predicted for the XY Bragg glass, $\delta=2.45$ [5, 34], shown by the dashed line in the figure. We note that like the XY Bragg glass phase, the smectic phase of pure liquid crystals possesses quasilong range order due to the Landau-Peierls instability and hence also displays a power-law singular scattering peak. However, the power-law exponent along $q_{\|}$for the smectic phase is always less than 2 [40, 41], a value that is incompatible with the measured line shapes for $8 \mathrm{CB}$ confined to aligned aerosil gels. In contrast, the close quantitative agreement between the exponent predicted for the XY Bragg glass and the observed values lends strong support to the conclusion that this topologically ordered phase forms in $8 \mathrm{CB}$ confined to aligned aerosil gels with low density.

Despite this excellent, quantitative agreement between the measured scattering profiles for low densities of aerosil and the XY Bragg glass form, we note that some caution is in order. As described previously, one can analyze the scattering intensity using Eq. (1) with $\xi_{2 \|}=\xi_{1 \|}$ and $\xi_{2 \perp}=\xi_{1 \perp}$. (Although, the agreement is not nearly as accurate as that of the XY Bragg glass form nor does it cover as large a wave-vector range; see Fig. 5(c) in Ref. [14]). Further, the values that are obtained for $\xi_{\|}$and $\xi_{\perp}$ from fitting to Eq. (1) are similar to those found for $8 \mathrm{CB}$ confined to isotropic aerosil gels following the same analysis [14]. As we argue above, this analysis provides a flawed physical picture; however, this coincidence in the correlation lengths suggests that the smectic correlations that form in the aligned gels and the isotropic gels are similar. Since the XY Bragg glass phase is not expected to be relevant to smectics confined to isotropic gels, one might interpret this similarity as 
evidence against the XY Bragg glass phase forming in the aligned gels. Additional experimental work on other smectic liquid crystals confined to aligned gels that test for the presence of the XY Bragg glass phase would help in clarifying the stability of this topologically ordered phase within the anisotropic random environment created by the gel.

\section{HIGH-TEMPERATURE CORRELATIONS: PSEUDO-CRITICAL BEHAVIOR}

As described above, the high-temperature scattering intensity of $8 \mathrm{CB}$ confined to aligned aerosil gels is characterized by dynamic critical fluctuations. We interpret these fluctuations as the remnants of the N-SmA critical point that is destroyed by the quenched disorder (and not directly with the transition to the XY Bragg glass phase which presumably has a significantly subtler signature in the $\mathrm{x}$-ray scattering than the observed robust peaks illustrated in Fig. 3). Although the N-SmA critical point is obtained strictly only in the presence of zero quenched disorder, upon cooling in weak disorder the system in some sense comes in close proximity to the critical point and should therefore display critical smectic fluctuations. Further, if the disorder is sufficiently weak, the susceptibility and correlation lengths charactering these fluctuations, while non-singular, should display pseudocritical behavior over an extended range of reduced temperature. This premise leads us to define the effective transition temperature $\mathrm{T}^{*}$ as the temperature at which these quantities would diverge if their growth were not truncated:

$$
\begin{aligned}
q_{0} \xi_{1 \|} & \sim|t|^{-\nu_{\|}} \\
q_{0} \xi_{1 \perp} & \sim|t|^{-\nu_{\perp}} \\
\sigma_{1} & \sim|t|^{-\gamma}
\end{aligned}
$$

where $t \equiv\left(\mathrm{T}-\mathrm{T}^{*}\right) / \mathrm{T}^{*}$ is the reduced temperature, and $\nu_{\|}, \nu_{\perp}$, and $\gamma$ are effective critical exponents for $\xi_{1 \|}, \xi_{1 \perp}$, and $\sigma_{1}$, respectively.

If this assumption of truncated power-law divergences is valid, then the three critical parameters should yield self-consistent values for $\mathrm{T}^{*}$. To test this assumption and to identify the correct value of $\mathrm{T}^{*}$, we fit the values of $\xi_{1 \|}$, $\xi_{1 \perp}$ and $\sigma_{1}$ to Eqs. (5)-(7). In these fits, only data at high temperatures where the dynamic term in Eq. (1) alone describes accurately the measured line shapes were included. As mentioned above, at lower temperature (near but below $\mathrm{T}^{*}$ ), additional contributions to the scattering intensity, presumably from the static correlations of the XY Bragg glass phase, appear. Since efforts to separate these different contributions lead to considerable uncertainties in the fit parameters, we focus on the high temperature side of the critical region. In the fitting, the critical exponents were held fixed and $\mathrm{T}^{*}$ was treated as a free parameter. These fits were repeated over a range of critical exponents, and the quality of the fits, as determined from the $\chi^{2}$ values, was compared. For all aerosil
TABLE I: Summary of effective critical exponents for $8 \mathrm{CB}$ confined to aligned aerosil gels in comparison with pure $8 \mathrm{CB}$ and the 3D XY model. Also included are the gel pore chords $l_{0}$ [19] and effective transition temperatures $\mathrm{T}^{*}$.

\begin{tabular}{|c||c|c|c|c|c|c|}
\hline System & $l_{0}(\AA)$ & $T^{*}(\mathrm{~K})$ & $\gamma$ & $\nu_{\|}$ & $\nu_{\perp}$ & $\nu_{\|} / \nu_{\perp}$ \\
\hline \hline Pure 8CB & & 306.97 & 1.26 & 0.67 & 0.51 & 1.314 \\
\hline $0.027 \mathrm{~g} / \mathrm{cm}^{3}$ & $\sim 3000$ & 305.96 & $\begin{array}{c}1.55 \\
\pm 0.05\end{array}$ & $\begin{array}{c}0.73 \\
\pm 0.02\end{array}$ & $\begin{array}{c}0.70 \\
\pm 0.02\end{array}$ & 1.05 \\
\hline $0.042 \mathrm{~g} / \mathrm{cm}^{3}$ & $\sim 1500$ & 305.01 & $\begin{array}{c}1.54 \\
\pm 0.05\end{array}$ & $\begin{array}{c}0.74 \\
\pm 0.03\end{array}$ & $\begin{array}{c}0.65 \\
\pm 0.03\end{array}$ & 1.13 \\
\hline $0.071 \mathrm{~g} / \mathrm{cm}^{3}$ & $\sim 900$ & 305.62 & $\begin{array}{c}1.56 \\
\pm 0.05\end{array}$ & $\begin{array}{c}0.75 \\
\pm 0.02\end{array}$ & $\begin{array}{c}0.66 \\
\pm 0.02\end{array}$ & 1.15 \\
\hline $0.10 \mathrm{~g} / \mathrm{cm}^{3}$ & $\sim 600$ & 305.86 & $\begin{array}{c}1.53 \\
\pm 0.05\end{array}$ & $\begin{array}{c}0.73 \\
\pm 0.02\end{array}$ & $\begin{array}{c}0.65 \\
\pm 0.03\end{array}$ & 1.13 \\
\hline $3 \mathrm{D} \mathrm{XY}$ & & & 1.32 & 0.67 & 0.67 & 1 \\
\hline
\end{tabular}

densities, the minima in $\chi^{2}$ for the three power-law relations, Eqs. (5)-(7), occur at values of $\mathrm{T}^{*}$ that are within $0.01 \mathrm{~K}$ of each other. We equate $\mathrm{T}^{*}$ with the average of the three values determined in this way for each aerosil density. This value is listed in Table I. Comparing $\mathrm{T}^{*}$ for different aerosil densities, we note the value displays a non-monotonic dependence on $\rho_{s}$ with a minimum near $\rho_{s}=0.05 \mathrm{~g} / \mathrm{cm}^{3}$, consistent with the effective critical temperature determined with calorimetry for $8 \mathrm{CB}$ confined to isotropic aerosil gels [19].

Figures 9 and 10 display log - log plots of $\xi_{1 \|}, \xi_{1 \perp}$ and $\sigma_{1}$ as a function of reduced temperature for $\mathrm{T}>\mathrm{T}^{*}$ for various gel densities using the optimal values of $\mathrm{T}^{*}$. The solid lines in the figures are the results of fits to Eqs. (5)(7), which demonstrate that the temperature dependence is well described by power laws, further reinforcing the idea of pseudo-critical behavior. The effective critical exponents extracted from the fits are listed in Table I along with the values for pure $8 \mathrm{CB}[35,36,37]$. The values of $\nu_{\|}, \nu_{\perp}$, and $\gamma$ for $8 \mathrm{CB}$ confined to aligned aerosil gels are distinctly different from those of pure $8 \mathrm{CB}$, indicating that the quenched disorder introduced by the gel not only affects the low-temperature smectic order but also modifies the pre-transitional critical fluctuations. At sufficiently high temperature the correlation lengths are small compared to the mean pore chord $l_{0}$ of the gels [19], which is also listed in Table I Hence, the disorder caused by the gels should have little direct effect on the smectic fluctuations well above $\mathrm{T}^{*}$. In the simplest picture of the $\mathrm{N}-\mathrm{SmA}$ transition, the formation of the smectic density wave breaks 3D XY symmetry, and thus the critical behavior of pure liquid crystals could be expected to match that of the 3D XY model. However, the observed critical behavior of pure $8 \mathrm{CB}$ and other smectic liquid crystals typically deviates from that of the 3D XY model. These deviations are illustrated by the difference in critical exponent values for pure $8 \mathrm{CB}$ and the $3 \mathrm{D} \mathrm{XY}$ model listed 

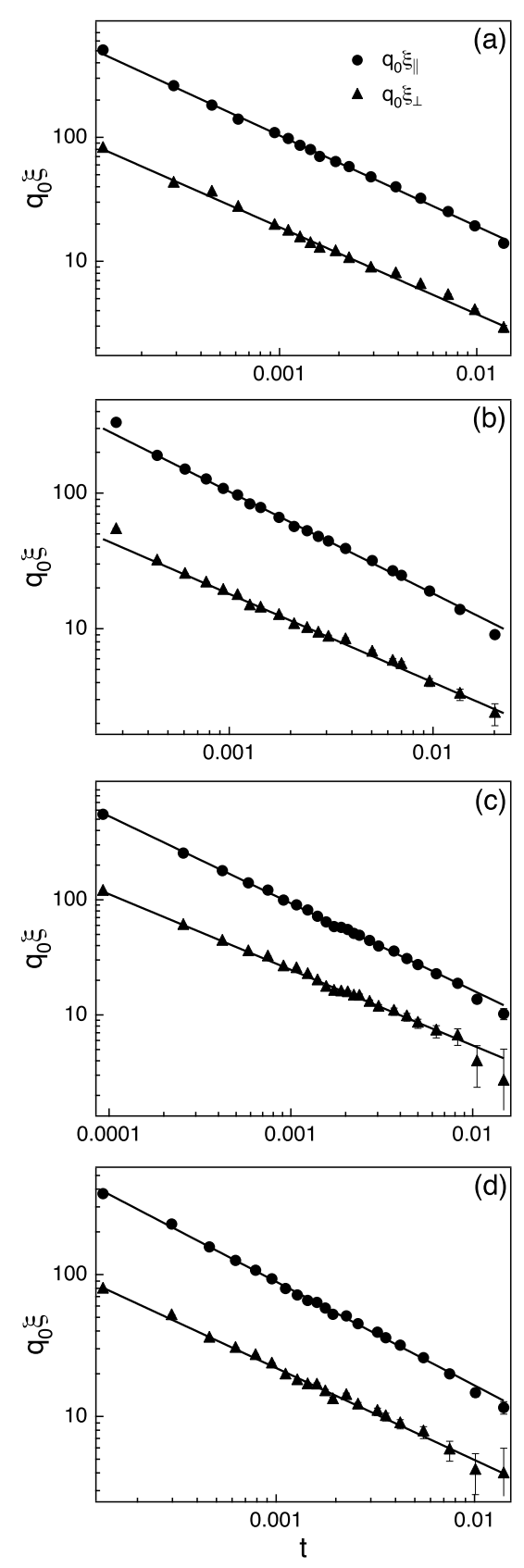

FIG. 9: Dynamic correlation lengths $\xi_{1 \|}$ (circles) and $\xi_{1 \perp}$ (triangles) as a function of reduced temperature for $8 \mathrm{CB}$ confined to aligned aerosil gels with densities (a) $\rho_{s}=0.027 \mathrm{~g} / \mathrm{cm}^{3}$, (b) $\rho_{s}=0.042 \mathrm{~g} / \mathrm{cm}^{3}$, (c) $\rho_{s}=0.071 \mathrm{~g} / \mathrm{cm}^{3}$, and (d) $\rho_{s}=0.10$ $\mathrm{g} / \mathrm{cm}^{3}$. The solid line are the results of power-law fits.

in Table The precise nature of these deviations, whose source likely involves couplings between the smectic order parameter and the nematic order, has been a longstanding problem. As described above, when a smectic liquid crystal is confined to an aligned aerosil gel, it experiences not only positional disorder that couples to the smectic density wave but also anisotropic orientational fields that couple to the nematic order, thereby modify-
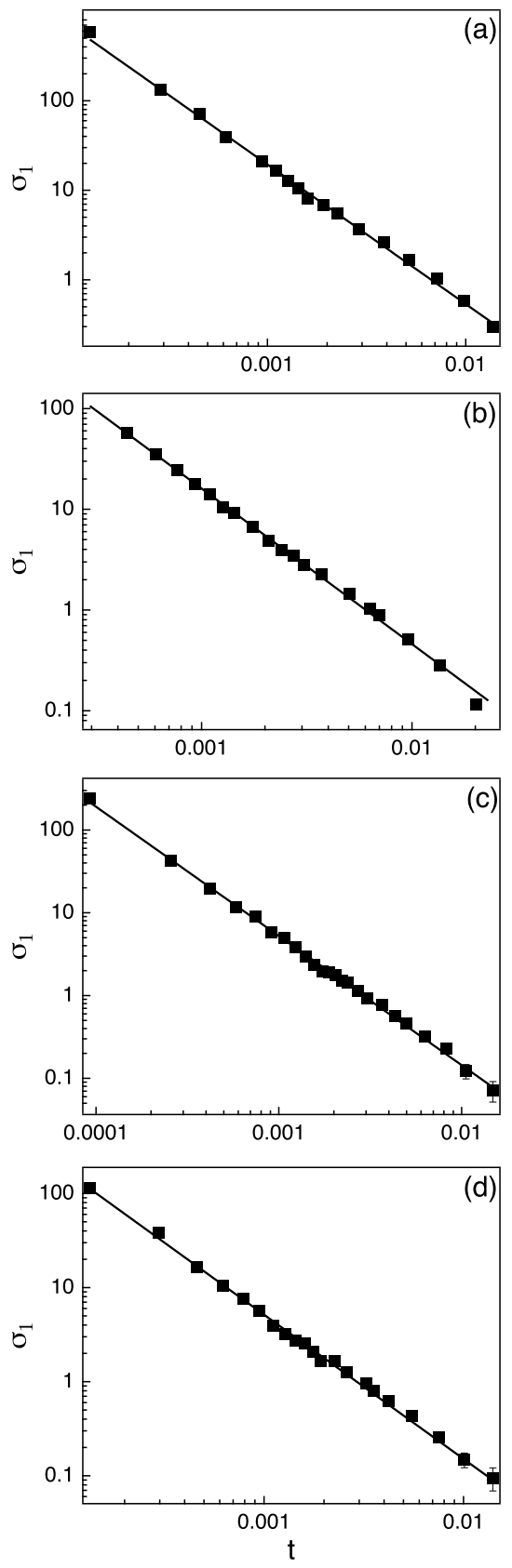

FIG. 10: Smectic susceptibilty as a function of reduced temperature for $8 \mathrm{CB}$ confined to aligned aerosil gels of density for (a) $\rho_{s}=0.027 \mathrm{~g} / \mathrm{cm}^{3}$, (b) $\rho_{s}=0.042 \mathrm{~g} / \mathrm{cm}^{3}$, (c) $\rho_{s}=0.071$ $\mathrm{g} / \mathrm{cm}^{3}$, and (d) $\rho_{s}=0.10 \mathrm{~g} / \mathrm{cm}^{3}$. The solid line are the results of power-law fits.

ing the nematic behavior. We therefore conclude that differences in the critical fluctuations between pure $8 \mathrm{CB}$ and $8 \mathrm{CB}$ confined to aerosil gels originate from the coupling of nematic order to the gel and its indirect influence on the smectic critical behavior.

As an illustration, compare the ratio $\nu_{\|} / \nu_{\perp}$ for varying aerosil densities with the ratio for pure $8 \mathrm{CB}$ listed in Table I] For the 3D XY model, the ratio is one due to 


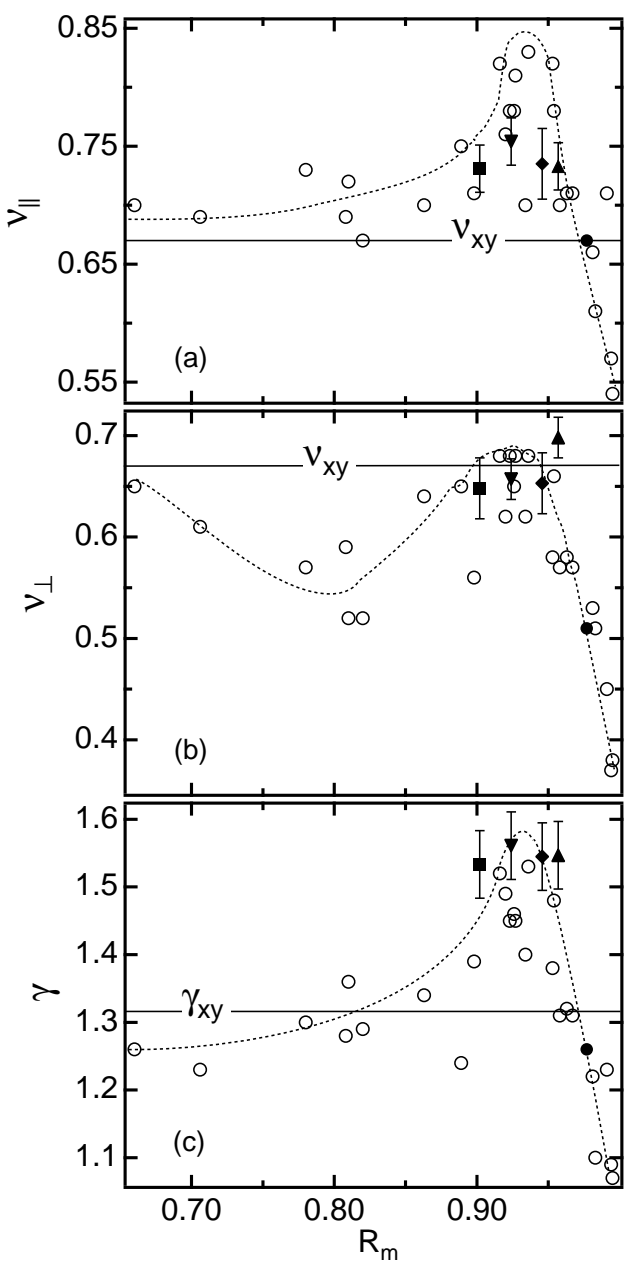

FIG. 11: Critical exponents (a) $\nu_{\|}$, (b) $\nu_{\perp}$, and (c) $\gamma$ for different smectic liquid crystals as a function of McMillan ratio, $R_{M} \equiv \frac{T_{N A}}{T_{N I}}$. The values for various pure liquid crystals, shown by circles, are taken from [42]. The values for pure $8 \mathrm{CB}$ are shown by the solid circles. Also included are the values of the effective critical exponents for $8 \mathrm{CB}$ confined to aligned aerosil gels with densities $\rho_{s}=0.027 \mathrm{~g} / \mathrm{cm}^{3}$, (triangle), $\rho_{s}=0.042$ $\mathrm{g} / \mathrm{cm}^{3}$ (diamond), $\rho_{s}=0.071 \mathrm{~g} / \mathrm{cm}^{3}$ (upside-down triangle), and $\rho_{s}=0.10 \mathrm{~g} / \mathrm{cm}^{3}$ (square), which are assigned effective McMillan ratios using Eq. (9). The solid lines mark the exponent values for the $3 \mathrm{D} \mathrm{XY}$ model, and the dashed lines are guides to the eye.

the isotropic scaling of correlations. However, for pure 8CB the scalings are highly anisotropic $\left(\nu_{\|} / \nu_{\perp}=1.314\right)$, consistent with behavior seen in various other smectic liquid crystals [42]. Patton and Andereck have advanced a theory that this anisotropic scaling results from coupling between nematic director fluctuations and the smectic order parameter 43, 44]. For 8CB confined to aligned aerosil gels, the ratio $\nu_{\|} / \nu_{\perp}$ shows much weaker scaling anisotropy than in pure $8 \mathrm{CB}$. One interpretation for this decreased scaling anisotropy is that pinning of the nematic director by the anisotropic orientational fields suppresses long-wavelength nematic fluctuations, thus weak- ening their influence over the smectic critical behavior.

While the decrease in $\nu_{\|} / \nu_{\perp}$ for $8 \mathrm{CB}$ confined to aligned aerosil gels compared with $\nu_{\|} / \nu_{\perp}$ for pure $8 \mathrm{CB}$ indicates qualitatively that the quenched disorder weakens nematic-smectic coupling, this observation would be made stronger with a quantitative measure of this weakening. In pure liquid crystals, the strength of the nematic-smectic coupling can be crudely parameterized by the McMillan ratio

$$
R_{M} \equiv \frac{T_{N A}}{T_{N I}}
$$

specifying the temperature range of the nematic phase. Large $R_{M}$ indicates a short nematic range, which typically implies the nematic order is far from saturated at the N-SmA transition. Hence, for large $R_{M}$ the nematic susceptibility can be expected to be large, and concomitantly the order parameter coupling can be expected to be strong. In a comprehensive survey of smectic critical behavior in pure liquid crystals, Garland and Nounesis found that the critical exponents $\alpha, \gamma, \nu_{\|}$, and $\nu_{\perp}$ obtained from calorimetry and x-ray scattering studies displayed complex but systematic trends as a function of $R_{M}$ [42]. Figures 11(a)-11(c) display the values of the critical exponents $\nu_{\|}, \nu_{\perp}$, and $\gamma$ of various smectic liquid crystals as a function of $R_{M}$, as originally assembled by Garland and Nounesis. Liquid crystals with small $R_{M}$ generally have critical exponents that approach the $3 \mathrm{D}$ $\mathrm{XY}$ values; however, the values of the exponents do not change monotonically with decreasing $R_{M}$. The critical exponents for pure $8 \mathrm{CB}\left(R_{M}=0.977\right)$ are shown by the solid circles in the figures. Also in Figs. 11(a)-11(c) are the effective critical exponents for $8 \mathrm{CB}$ confined to aligned aerosil gels, which have been placed on the figures by assigning the smectics with quenched disorder an effective McMillan ratio,

$$
R_{M}^{e f f}=0.977-0.72 \rho_{s}
$$

As the figures illustrate, this mapping between disorder strength, as parameterized by aerosil density, and nematic-smectic coupling, as parameterized by McMillan ratio, places the effective critical exponents for $8 \mathrm{CB}$ confined to aligned aerosil densities roughly in line with trends of pure liquid crystals of varying $R_{M}$. We note a very similar linear mapping of aerosil density to effective McMillan ratio was introduced previously for $8 \mathrm{CB}$ and 40.8 confined to isotropic aerosil gels [12, 28]. In these cases, the mapping was shown to collapse successfully effective heat capacity and order parameter exponents, $\alpha$ and $\beta$, for various aerosil densities onto the trends of pure liquid crystals. The linear coefficient that optimized the mapping for the smectics confined to isotropic aerosil gels was smaller than that in Eq. (9), 0.47 as opposed to 0.72 , suggesting that aligned aerosil gels are more effective than isotropic gels in suppressing nematic-smectic order parameter coupling. Nevertheless, these linear mappings provide a quantitative measure of how the presence 
of the quenched disorder influences the smectic critical behavior. The results in Figs. 11(a)-11(c) thus extend the notion of an effective McMillan ratio to the effective critical exponents $\nu_{\|}, \nu_{\perp}$, and $\gamma$, making comprehensive the analogy between disorder strength and $R_{M}^{e f f}$ for describing the behavior of the pre-transitional critical fluctuations of smectic confined to aerosil gels. Further theoretical efforts to understand the effects of quenched disorder could hence be a fruitful avenue for unraveling the outstanding mysteries surrounding the critical behavior of pure smectics.
Acknowledgements: We gratefully acknowledge C. Garland, G. Iannacchione, B. Ocko, and L. Radzihovsky for helpful discussions. Funding was provided by the NSF under Grant No. DMR-0134377. Use of the National Synchrotron Light Source, Brookhaven National Laboratory, was supported by the U. S. Department of Energy, Office of Science, Office of Basic Energy Sciences, under Contract No. DE-AC02-98CH10886.
[1] L. Radzihovsky and J. Toner, Phys. Rev. B 60, 206 (1999).

[2] L. Radzihovsky and J. Toner, Phys. Rev. Lett. 78, 4414 (1997).

[3] L. Radzihovsky and J. Toner, Phys. Rev. Lett. 79, 4214 (1997).

[4] B. Jacobsen, K. Saunders, L. Radzihovsky, and J. Toner, Phys. Rev. Lett. 83, 1363 (1999).

[5] K. Saunders, PhD thesis, University of Oregon, 2001.

[6] T. Bellini, L. Radzihovsky, J. Toner, and N. A. Clark, Science 294, 1074 (2001).

[7] L. Wu, B. Zhou, C. W. Garland, T. Bellini, and D. W. Schaefer, Phys. Rev. E 51, 2157 (1995).

[8] H. Zeng, B. Zalar, G. S. Iannacchione, and D. Finotello, Phys. Rev. E 60, 5607 (1999).

[9] T. Bellini, N. A. Clark, and D. W. Schaefer, Phys. Rev. Lett. 74, 2740 (1995).

[10] S. Park et al., Phys. Rev. E 65, 050703 (2002).

[11] T. Jin and D. Finotello, Phys. Rev. Lett. 86, 818 (2001).

[12] R. L. Leheny et al., Phys. Rev. E 67, 011708 (2003).

[13] G. S. Iannacchione, S. Park, C. W. Garland, R. J. Birgeneau, and R. L. Leheny, Phys. Rev. E 67, 011709 (2003).

[14] D. Liang, M. A. Borthwick, and R. L. Leheny, J. Phys. Condens. Matter 16, S1989 (2004).

[15] T. Jin and D. Finotello, Phys. Rev. E 69, 041704 (2004).

[16] B. Zhou, G. S. Iannacchione, C. W. Garland, and T. Bellini, Phys. Rev. E 55, 2962 (1997).

[17] A. Mertelj, A. Jákli, and M. Čopič, Mol. Cryst. Liq. Cryst. 331, 1941 (1999).

[18] A. Hourri, T. K. Bose, and J. Thoen, Phys. Rev. E 63, 051702 (2001).

[19] G. S. Iannacchione, C. W. Garland, J. T. Mang, and T. P. Rieker, Phys. Rev. E 58, 5966 (1998).

[20] G. Cordoyiannis, S. Kralj, G. Nounesis, S. Žumer, and Z. Kutnjak, Phys. Rev. E 73, 031707 (2006).

[21] A. Roshi, G. S. Iannacchione, P. S. Clegg, R. J. Birgeneau, and M. E. Neubert, Phys. Rev. E 72, 051716 (2005).

[22] G. Sinha, J. Leys, C. Glorieux, and J. Thoen, Phys. Rev.
E 72, 051710 (2005).

[23] F. Mercuri, S. Paoloni, U. Zammit, and M. Marinelli, Phys. Rev. Lett. 94, 247801 (2005).

[24] J. Leys, G. Sinha, C. Glorieux, and J. Thoen, Phys. Rev. E 71, 051709 (2005).

[25] M. Caggioni et al., Phys. Rev. Lett. 93, 127801 (2004).

[26] P. S. Clegg et al., Phys. Rev. E 68, 031706 (2003).

[27] P. S. Clegg et al., Phys. Rev. E 67, 021703 (2003).

[28] S. Larochelle, M. Ramazanoglu, and R. J. Birgeneau, Phys. Rev. E 73, 060702 (2006).

[29] R. Bandyopadhyay, D. Liang, R. H. Colby, J. L. Harden, and R. L. Leheny, Phys. Rev. Lett. 94, 107801 (2005).

[30] T. Giamarchi and P. L. Doussal, Phys. Rev. Lett. 72, 1530 (1994).

[31] M. J. P. Gingras and D. A. Huse, Phys. Rev. B 53, 15193 (1996).

[32] D. S. Fisher, Phys. Rev. Lett. 78, 1964 (1997).

[33] M. Tissier and G. Tarjus, Phys. Rev. Lett. 96, 087202 (2006).

[34] L. Chen and J. Toner, Phys. Rev. Lett. 94, 137803 (2005).

[35] B. M. Ocko, R. J. Birgeneau, and J. D. Litster, Z. Phys. B 62, 487 (1986).

[36] J. Thoen, H. Marynissen, and W. V. Dael, Phys. Rev. A 26, 2886 (1982).

[37] D. Davidov et al., Phys. Rev. B 19, 1657 (1979).

[38] T. Bellini, N. A. Clark, V. Degiorgio, F. Mantegazza, and G. Natale, Phys. Rev. E 57, 2996 (1998).

[39] P. Dutta and S. K. Sinha, Phys. Rev. Lett. 47, 50 (1981).

[40] V. M. Kaganer, B. I. Ostrovskii, and W. H. de Jeu, Phys. Rev. A 44, 8158 (1991).

[41] J. Als-Nielsen et al., Phys. Rev. B 22, 312 (1980).

[42] C. W. Garland and G. Nounesis, Phys. Rev. E 49, 2964 (1994).

[43] B. R. Patton and B. S. Andereck, Phys. Rev. Lett. 69, 1556 (1992).

[44] B. S. Andereck and B. R. Patton, Phys. Rev. E 49, 1393 (1994). 